\title{
Essential role of the CUL4B ubiquitin ligase in extra-embryonic tissue development during mouse embryogenesis
}

\author{
Liren Liu ${ }^{1}$, Yan Yin ${ }^{2}$, Yuewei $\mathrm{Li}^{1}$, Lisa Prevedel ${ }^{1}$, Elizabeth H Lacy ${ }^{3}$, Liang $\mathrm{Ma}^{2}$, Pengbo Zhou ${ }^{1}$ \\ ${ }^{I}$ Department of Pathology and Laboratory Medicine, Weill Cornell Medical College and Weill Graduate School of Medical Sci- \\ ences of Cornell University, New York, NY 10065, USA; ${ }^{2}$ Division of Dermatology, Washington University School of Medicine, St. \\ Louis, MO 63110, USA; ${ }^{3}$ Developmental Biology Program, Memorial Sloan-Kettering Cancer Center, New York, NY 10065, USA
}

\begin{abstract}
Mutations of the $C U L 4 B$ ubiquitin ligase gene are causally linked to syndromic X-linked mental retardation (XLMR). However, the pathogenic role of CUL4B mutations in neuronal and developmental defects is not understood. We have generated mice with targeted disruption of $C u l 4 b$, and observed embryonic lethality with pronounced growth inhibition and increased apoptosis in extra-embryonic tissues. $\mathrm{Cul4b}$, but not its paralog $\mathrm{Cul4a}$, is expressed at high levels in extra-embryonic tissues post implantation. Silencing of CUL4B expression in an extra-embryonic cell line resulted in the robust accumulation of the CUL4 substrate $221^{\mathrm{Cip} 1 / \mathrm{WAF}}$ and G2/M cell cycle arrest, which could be partially rescued by silencing of $p 21^{C i p / W A F}$. Epiblast-specific deletion of $C u l 4 b$ prevented embryonic lethality and gave rise to viable $C u l 4 b$ null mice. Therefore, while dispensable in the embryo proper, $C u l 4 b$ performs an essential developmental role in the extra-embryonic tissues. Our study offers a strategy to generate viable $\mathrm{Cul}$ (4b-deficient mice to model the potential neuronal and behavioral deficiencies of human CUL4B XLMR patients.
\end{abstract}

Keywords: cullin 4B; knockout; extra-embryonic tissue; ubiquitin; X-inactivation

Cell Research (2012) 22:1258-1269. doi:10.1038/cr.2012.48; published online 27 March 2012

\section{Introduction}

Mental retardation (MR) is a disability characterized by limitations of intellectual and adaptive skills. To date, over 900 genetic mutations are linked to MR, highlighting the enormous heterogeneity of this clinical condition [1]. Of the known genetic lesions, the X-chromosome harbors over $100 \mathrm{MR}$ genes, far more than those found on autosomes. Recently, multiple mutations of the human CUL4B ubiquitin ligase gene (Xq24) were identified as being causally associated with X-linked MR (XLMR) [2-5]. CUL4B-deficient patients display clinical symptoms that include growth retardation, macrocephaly, hypogonadism, obesity, and ataxia [2-5].

Correspondence: Pengbo Zhou

Tel: +1-212-746-6415; Fax: +1-212-746-4483

E-mail: pez2001@med.cornell.edu

Received 1 December 2011; revised 8 January 2012; accepted 21 February 2012; published online 27 March 2012
CUL4B is a member of the cullin-RING ubiquitin ligase (CRL) family, which is the largest E3 ligase subtype in mammals that controls a wide spectrum of cellular processes including cell cycle, DNA replication and repair, signaling, transcription, and embryonic development (reviewed in [6-8]). CRLs are multimeric E3 ligase complexes that assemble on the elongated cullin scaffold: the RING domain adaptor Rbx1/Roc1 recruits the E2 ubiquitin-conjugating enzyme and interacts with the C-terminal cullin homology domain, while the cullin $\mathrm{N}$-terminus associates with a cullin-specific adaptor protein to recruit a large family of substrate receptors, each of which can bind multiple substrates. The CUL4 family is unique among the eight cullins as there are two CUL4 members, CUL4A and CUL4B, that share about $82 \%$ sequence identity. CUL4A and CUL4B assemble structurally similar E3 complexes using the same adaptors (Rbx1 and DDB1) and DCAF substrate receptors, and function redundantly to target critical cell cycle regulators, chromatin modifiers and transcription factors for ubiquitination and degradation, thereby supporting growth and sur- 
vival (reviewed in $[8,9]$ ). Among the CUL4 substrates that control cell cycle progression, the cyclin-dependent kinase inhibitor p21 was recently shown to be targeted for ubiquitination and degradation by both CUL4A and CUL4B under normal conditions as well as post-UV irradiation in cell lines $[10,11]$. However, primary mouse embryonic fibroblasts preferentially utilize CUL4A to restrict p21 levels [12], suggesting context-dependent degradation of p21 by the two CUL4 ubiquitin ligases. Cul4a null mice are viable and display no gross phenotypic abnormalities throughout their lifespan except for meiotic defects in male mice [12-14]. Despite the largely overlapping functions of CUL4A and CUL4B, recent studies also revealed distinct roles for the two CUL4 family members in DNA-damage response, male meiosis, and response to environmental toxins [12-15]. While CUL4B compensates for the loss of CUL4A [12], CUL4A expression, which is unaffected by CUL4B mutations [16], is apparently unable to rescue the neuronal and developmental deficiencies found in $C U L 4 B$ patients with XLMR. Notably, the loss of CUL4B function is not lethal in humans [2-5].

Like human $C U L 4 B$, the mouse $C u l 4 b$ gene also resides on X-chromosome. Here, we generated the $\mathrm{Cul} 4 \mathrm{~b}$ knockout mice to determine the physiological functions of CUL4B and the pathological basis of CUL4B deficiency in XLMR and the associated developmental defects. While human $C U L 4 B$ patients can survive to adulthood, Cul4b knockout mice die as early as E7.5, consistent with the observed embryonic lethality upon inactivation of $C u l 4 b$ by gene trapping [17]. The developmental defects of $C u l 4 b$ null embryos were attributed exclusively to the degeneration of extra-embryonic tissues, while the epiblast remained largely unaffected by $C u l 4 b$ deletion. Embryos with conditional deletion of $C u l 4 b$ in the epiblast were viable and displayed no overt developmental defects compared to their wild-type littermates. Our results reveal an essential developmental role of CUL4B in the extra-embryonic tissues and highlight the differential requirement between mice and humans for CUL4B during embryogenesis.

\section{Results}

\section{Generation of Cul4b null mice}

To investigate the physiological role of CUL4B, we generated conditional $C u l 4 b$ knockout mice using the Cre/lox strategy. Exons 4 and 5, which span the DDB1binding site [18], were floxed by loxP homologous recombination sites in embryonic stem (ES) cells (Figure 1A). Deletion of exons 4-5 by Cre recombinase removes the DDB1-binding site in the $\mathrm{N}$ terminus of CUL4B.
Moreover, splicing of exon 3 to 6 in the recombined $\mathrm{Cul} 4 \mathrm{~b}$ transcript would result in a reading-frame shift and a premature stop codon, thus generating a putative 78-amino acid fragment of CUL4B lacking both the DDB1-binding domain and the cullin homology domain that is necessary for Rbx1 binding and subsequent recruitment of the E2 ubiquitin-conjugating enzyme. The targeting event was confirmed by Southern blotting using probes external to the floxed $C u l 4 b$ sequences (Figure 1B). Mouse ES cells bearing floxed $C u l 4 b$ were microinjected into blastocysts to derive chimeras from which germline transmission of the mutant allele was achieved. Hemizygous $C u l 4 b^{f / Y}$ and homozygous $C u l 4 b^{f f f}$ mice were healthy and phenotypically indistinguishable from their wild-type littermates. $C u l 4 b^{f / Y}$ and $C u l 4 b^{f / f}$ mice were maintained on a C57BL/6J-129 mixed background and their offspring were genotyped by PCR (Figure 1C, left panel). $C u l 4 b^{f /+}$ mice subsequently interbred to $\mathrm{Vav}-\mathrm{iCre}$ mice that express the Cre recombinase in hematopoietic stem cells [19]. We validated the resulting hematopoietic-specific null allele of $C u l 4 b$ by PCR-based genotyping of genomic DNA from peripheral blood cells from the offspring of the $\mathrm{Cul}_{4} \mathrm{f}^{\mathrm{ft+}} \times \mathrm{Vav}-\mathrm{iCr} \mathrm{Cr}^{+/-}$mating (Figure 1C, right panel).

\section{Embryonic lethality of $\mathrm{Cul}_{4} \mathrm{~b}^{-/ Y}$ mice}

To model XLMR patients with CUL4B deficiency in mice, we initially attempted to generate $C u l 4 b$ null animals by crossing the floxed mice with EIIA-Cre transgenic mice [20]. This approach was unsuccessful due to the mosaic pattern of expression from the EIIA promoter (see Supplementary information, Data S1 and Table $\mathrm{S} 1)$. To bypass the mosaicism inherent to the EIIA-Cre mice, heterozygous $C u l 4 b^{f /+}$ female mice were crossed to chicken-actin (CAG)-Cre mice that drive Cre-mediated recombination at the early zygote stage [21]. As shown in Table 1, no viable hemizygous $C u l 4 b^{-/ Y}$ pups were generated among a total of 77 offspring from the mating between male $\mathrm{CAG}-\mathrm{Cr} e^{+/-}$and female $\mathrm{Cul}_{4} b^{f /+}$ mice. Hemizygous $\mathrm{Cul}_{4 b^{-/ Y}}$ embryos were still present at days E7.5 and E8.5 while no $C u l 4 b^{-/ Y}$ embryos were identified beyond E9.5, suggesting that $C u l 4 b$ null embryos lost viability prior to day E9.5 (Table 1).

Notably, the heterozygous $C u l 4 b^{+/}$embryos were also not viable, but they exhibited a less severe phenotype than the $C u l 4 b^{-/ Y}$ embryos: among a total of 40 embryos dissected at E9.5-E12.5, six were $C u l 4 b^{+/-}$heterozygotes (Table 1). At day E12.5, the yolk sacs of $C u l 4 b^{+-}$embryos had underdeveloped branching vitelline vessels compared to controls (Figure 2A), suggesting an insufficient supply of nutrients to the developing embryo. No $C u l 4 b^{+/-}$embryos were identified beyond E13.5. Since 
A

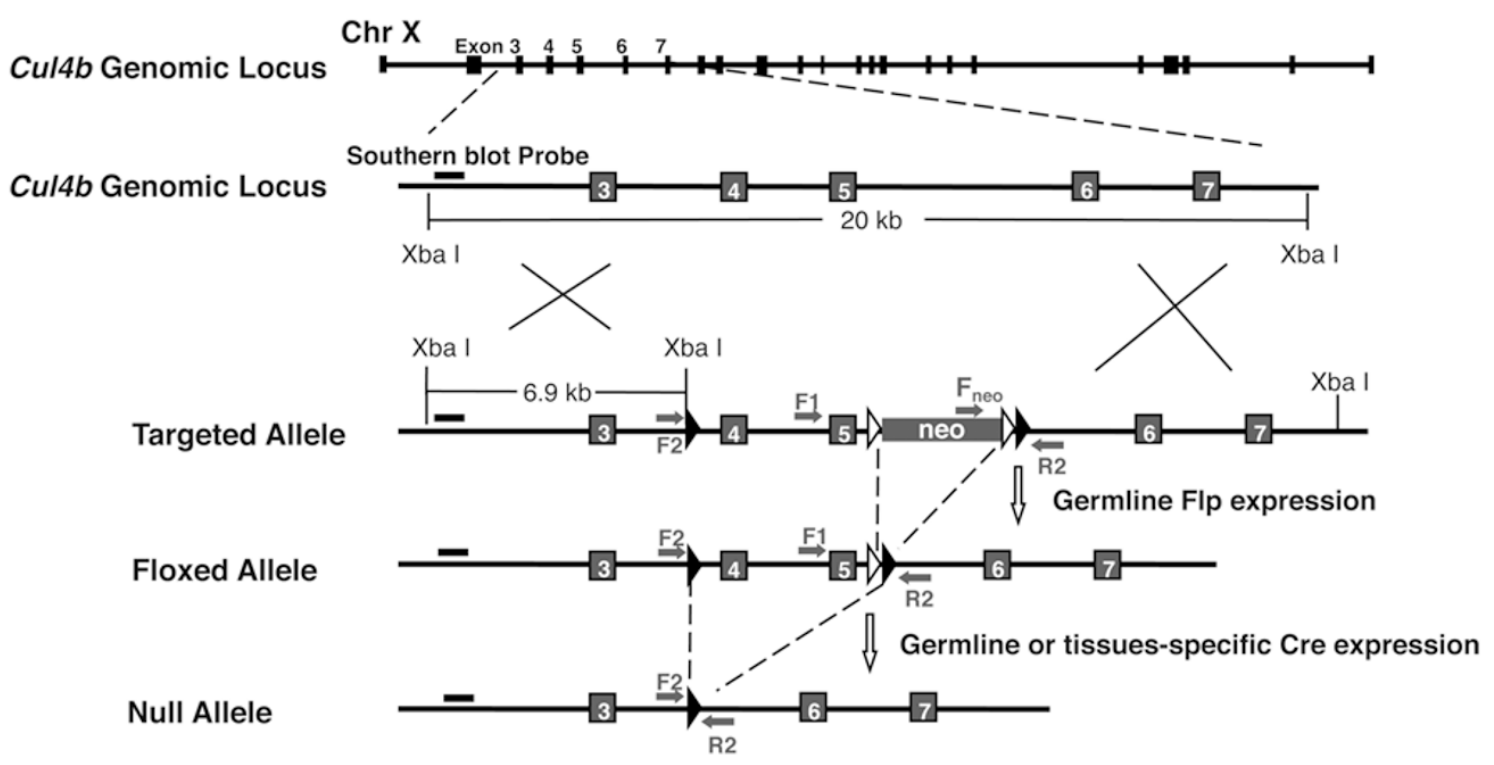

B
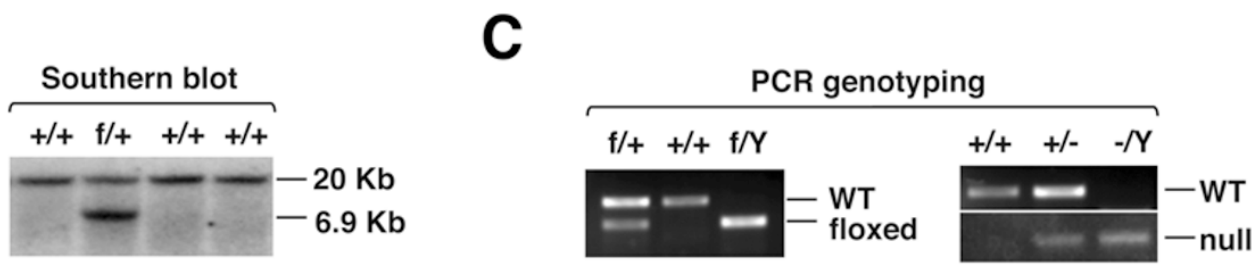

Figure 1 Generation of floxed and knockout alleles of Cul $4 b$ in mice. (A) Genomic structure of the Cul $4 b$ gene on the $X$-chromosome and the targeted alleles. LoxP sites (filled arrowheads) were introduced into the intron regions flanking exons 4-5 of the targeting construct for homologous recombination in ESCs. Frt recombination sites (empty arrowheads) were engineered for removal of the neomycin selection marker by germline Flp expression following crossing to ACT-FLPe transgenic mice. Arrows, PCR primers for genotyping; Bars, probe for Southern blotting. (B) Generation of targeted ES cells. G418resistant ES cell clones were screened by Southern blot analysis. DNA was digested with the Xbal restriction enzyme and hybridized to the probes as indicated in A. +/+, wild-type; f/+, floxed heterozygous allele. (C) PCR genotyping of the indicated alleles using tail DNA from wild-type to $\mathrm{Cul} 4 b^{f /+}$ breeding (left panel) or peripheral blood cell DNA from Vav-iCre to Cul $4 b^{f /+}$ breeding (right panel).

Table 1 Genotype analysis of E7.5-E12.5 embryos and 2-week pups from matings between $\mathrm{CAG}-\mathrm{Cre}\left({ }^{\AA}\right)$ and $\mathrm{Cul}_{4} \mathrm{~b}^{\mathrm{f/+}}\left({ }_{(}\right)$

\begin{tabular}{lcccc}
\hline Age & \multicolumn{3}{c}{ Genotype } & Total \\
\cline { 2 - 4 } & $\mathrm{Cul4b}^{\text {wt }}$ & $\mathrm{Cul4b}^{+/-}$ & $\mathrm{Cul4b}^{-/ Y}$ & \\
\hline E7.5 & 25 & 6 & 4 & 35 \\
E8.5 & 50 & 12 & 5 & 67 \\
E9.5-12.5 & 34 & 6 & 0 & 40 \\
2 weeks & 77 & 0 & 0 & 77 \\
\hline
\end{tabular}

the paternal $\mathrm{X}$ chromosome undergoes imprinted inactivation in the extra-embryonic tissues in mice [22], the $C u l 4 b^{+}$allele in the $C u l 4 b^{+/}$heterozygous embryos is transcriptionally silenced, accounting for their lost vi- ability (Supplementary information, Figure S1). It is noteworthy that $\mathrm{Cul}_{4} \mathrm{~b}^{+/-}$embryos survived an additional 2-3 days compared to the $C u l 4 b^{-/ Y}$ hemizygous embryos. Incomplete (or escape from) X-chromosome inactivation may have produced residual CUL4B protein, which may account for the limited proliferation and survival of $\mathrm{Cul}_{4 b^{+/}}$embryos prior to E12.5 [23, 24]. Another possible explanation is that the genes on the paternal $\mathrm{X}$ chromosome are transcriptionally active at the two-cell stage and gradually silenced by imprinted $\mathrm{X}$-inactivation as pre-implantation development continues [25]. As such, $C u l 4 b^{+/-}$embryos likely express a limited amount of CUL4B in the zygote whereas $C u l 4 b^{-/ Y}$ embryos do not.

While we were unable to recover live heterozygous $\mathrm{Cul}_{4} \mathrm{~b}^{+/-}$pups from the matings between male $C A G$ - 
A

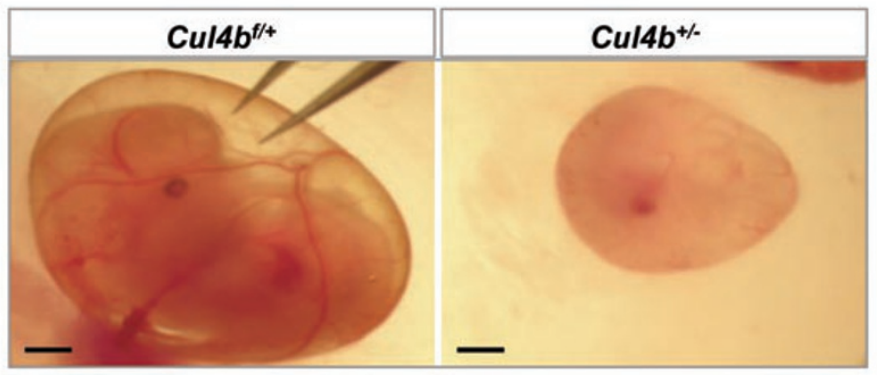

B
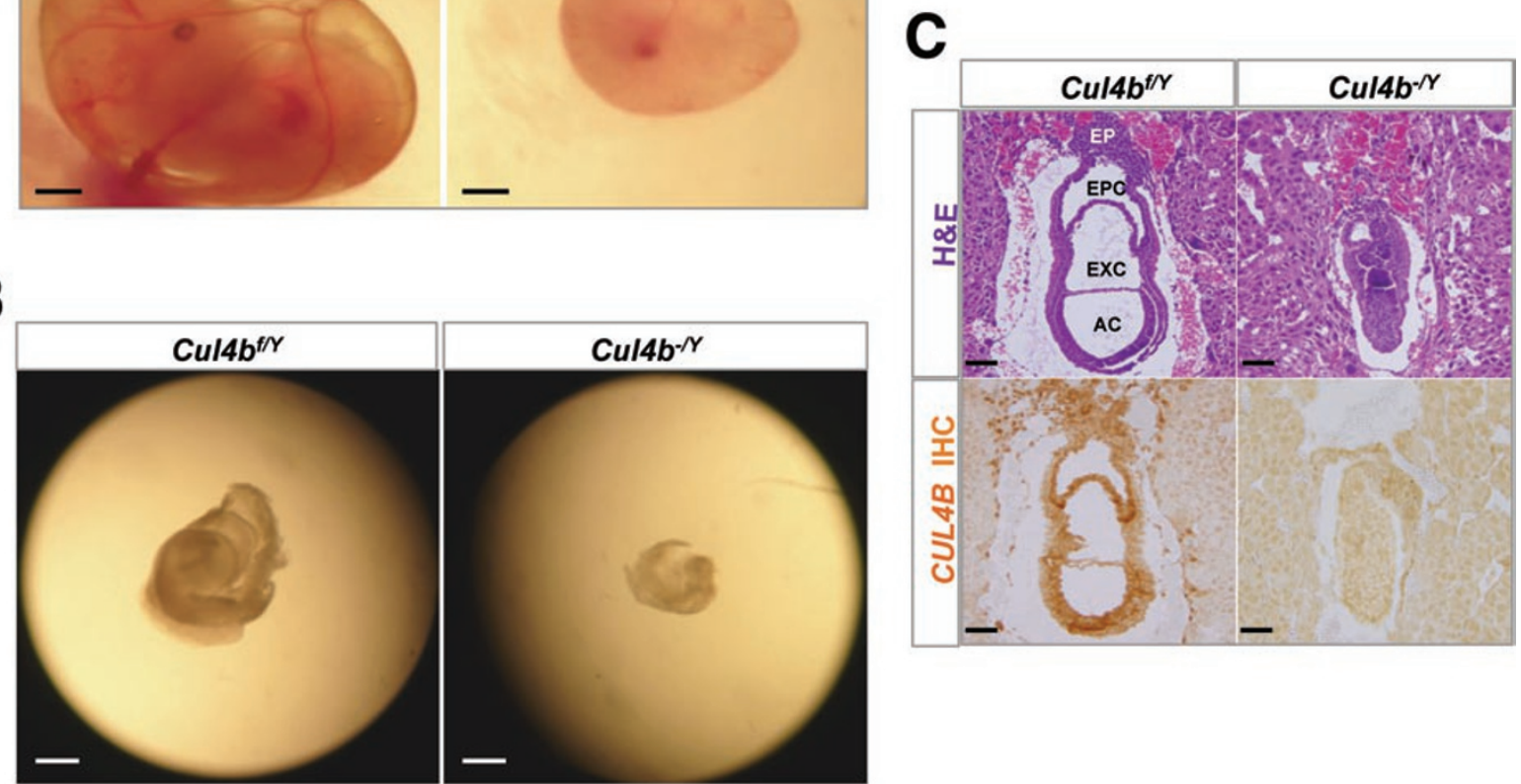

Figure 2 Cul $4 b$ deletion resulted in embryonic lethality. (A) Angiogenic defects of the Cul $4 b^{-/ \gamma}$ yolk sac. Note the poor vasculature and lack of branching vitelline vessels in $C u l 4 b^{-/ Y}$ yolk sac compared with those in the Cul4b $b^{f / Y}$ embryos at day E12.5. (B) Morphology of the dissected E8.5 embryos from Cul4 $b^{f / \gamma}$ and CAG-Cre mating. (C) Histological and immunohistochemical analysis of sagittal sections of E7.5 $\mathrm{Cu}_{4} 4 \mathrm{~b}^{-\gamma}$ and control Cul $4 b^{f / Y}$ embryos. AC: amniotic cavity; EXC: exocoelom cavity; EPC: ectoplacental cavity. Bars: $1.0 \mathrm{~mm}, 0.2 \mathrm{~mm}$ and $50 \mu \mathrm{m}$ in A, B, and C, respectively.

Table 2 Crossing directions between $C A G-C r e^{+/-}$and $C u l 4 b$ mutant mice affect productivity of the females and genotype of the offspring

\begin{tabular}{|c|c|c|c|c|c|c|}
\hline Male & Female & $\mathrm{Cul}_{4 b^{+/-}}$ & $\mathrm{Cult}^{-/ Y}$ & No. of litters & No. of pups & Average litter size \\
\hline $\mathrm{CAG}-\mathrm{Cr}^{+/-}$ & $C u l 4 b^{\mathrm{f} /+}$ & 0 & 0 & 19 & 77 & 4.1 \\
\hline $\mathrm{CAG}-\mathrm{Cre}^{+/-}$ & $\mathrm{Cul}_{4} \mathrm{f}^{\mathrm{f} / \mathrm{f}}$ & 0 & 0 & 6 & 17 & 2.8 \\
\hline$C u l 4 b^{\mathrm{f} / \mathrm{Y}}$ & CAG-Cre $e^{+-}$ & 23 & NA & 9 & 77 & 8.6 \\
\hline$C 57 B L / 6 J$ & $\mathrm{Cul}_{4 b^{+/-}}$ & 0 & 0 & 17 & 44 & 2.6 \\
\hline
\end{tabular}

Cre and female $C u l 4 b^{f f f}$ mice, the reciprocal cross (male

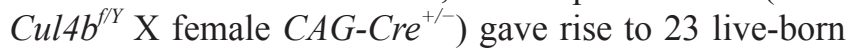
$C u l 4 b^{+-}$mice in a total of 77 pups from 9 matings (Table 2). These results indicate that the $C u l 4 b$ null allele cannot be transmitted from the mother to the next generation, in contrast to human $C U L 4 B$ patients who inherit X-linked $C U L 4 B$ mutations maternally. Given that the paternallyinherited $\mathrm{X}$ chromosome is inactivated in murine extraembryonic tissues [22], $\mathrm{Cul}_{4 b^{+/-}}$embryos bearing the maternal mutant allele are deficient for CUL4B ubiquitin ligase activity in the extra-embryonic lineages (Supple- mentary information, Figure S1). The resulting extraembryonic tissue defects likely account for the inability of heterozygous females to transmit the $C u l 4 b$ null allele to their offspring.

To evaluate the gross morphological changes in $C u l 4 b^{-/ Y}$ embryos, a litter of E8.5 embryos were dissected from pregnant $C u l 4 b^{f f f}$ mice bred with $C A G$-Cre mice. Although all the deciduas were of normal appearance and size (Supplementary information, Figure S2A and 2B), 2 out of 11 embryos showed severe morphological abnormalities and were dramatically reduced in size 
(Figure 2B, Supplementary information, Figure S2C). PCR genotyping confirmed that these abnormal embryos were indeed $C u l 4 b^{-/ Y}$ hemizygous knockouts (Supplementary information, Figure S2D). Notably, heterozygous $\mathrm{Cul}_{4} \mathrm{~b}^{+/-}$embryos at this stage were normal-sized and morphologically indistinguishable from the wildtype embryos.

The E7.5 deciduas were dissected and embedded for histological analysis. H\&E staining showed normal developmental appearance of wild-type embryos with the standard formation of the amniotic cavity, exocoelom, and ectoplacental cavities. Primitive streaks composed of different tissue layers were also readily recognizable in these embryos (Figure 2C). In contrast, $C u l 4 b^{-/ Y}$ embryos displayed compact, indiscernible structures, underdeveloped cavities, and absent ectoplacental cones (EPCn) (Figure 2C). Primitive streak layers were absent in these embryos, and the embryonic ectoderm was disorganized and reduced in size. Collectively, these data indicate that $C u l 4 b$ is essential for embryonic development.

\section{Growth arrest and degeneration of Cul4b null embryos}

To explore the underlying cause of embryonic lethality of $C u l 4 b^{-/ Y}$ mice, we evaluated cell proliferation and apoptosis in these mutants. 5-bromo-2'-deoxyuridine (BrdU) labeling revealed dramatically reduced numbers of proliferating cells throughout the E7.5 $\mathrm{Cul}_{4} \mathrm{~b}^{-/ Y}$ conceptuses (Figure 3A, white arrows). Notably, far fewer proliferating cells were detected in the placental cone (black arrow) and trophectoderm-derived tissues (Figure $3 \mathrm{C}$, compared to $3 \mathrm{~A}$ and $3 \mathrm{~B}$ ). At this stage, no difference in BrdU labeling was detected between wild-type and heterozygous conceptuses (Figure 3A and 3B). On the other hand, immunofluorescent (IF) staining of phosphoHistone H3 (PHH3), a well-established mitotic marker, revealed only a few mitotic cells in control embryos, whereas a marked increase in this cell population was evident in $\mathrm{Cul}_{4} b^{-/ Y}$ embryos (Figure 3D-3F). Considering the drastic decrease in the proliferation of $\mathrm{Cul}_{4} \mathrm{~b}^{-/ Y}$ extraembryonic cells (Figure 3A-3C), accumulation of PHH3positive cells indicates that these mutant cells have arrested at G2/M. Furthermore, TUNEL assays revealed increased apoptosis in both the embryonic and extraembryonic ectoderms of $C u l 4 b^{-/ Y}$ embryos, while very few TUNEL-positive cells were visible in the $C u l 4 b^{+/+}$ or $\mathrm{Cul}_{4 b^{+/}}$embryos (Figure 3G-3I). Together, these data indicate that CUL4B is essential for maintaining growth and survival during embryogenesis.

Dynamic expression of Cul4b and Cul4a during early embryonic development

While we previously showed that $C u l 4 b$ compensates for the loss of Cul4a in Cul4a $a^{-/-}$mice [12], the embryonic lethality of $C u l 4 b^{-/ Y}$ mice indicates that $C u l 4 a$ is unable to rescue $C u l 4 b$ deficiency. We set out to investigate whether the lethality of $C u l 4 b^{-1 Y}$ mice results from the differential expression patterns of the two CUL4 proteins, which were recently demonstrated as the cause of the meiotic defects in Cul4a $a^{-/-}$male mice $[13,14]$. In unhatched E3.5-4.0 blastocysts, both CUL4 proteins were detected in the inner cell mass (ICM) as well as in trophoblast (TB) cells (open arrowheads, Figure 4A-4D). However, CUL4B was highly expressed in the secondary polar body, where CUL4A was absent. In peri-implantation blastocysts (E4.5), both CUL4 proteins were present at comparable levels in the cytoplasm of most ICM and TB cells (Figure $4 \mathrm{~A}^{\prime}-4 \mathrm{D}^{\prime}$, open arrowheads). However, prominent nuclear CUL4B staining was evident in some TB cells (Figure 4B', inset, arrowheads). Why these cells exhibit differential staining patterns and whether CUL4B exerts different functions in these cells remain unclear. Upon implantation, the two CUL4 proteins continue to exhibit differential expression patterns. At E5.0, CUL4B was predominantly expressed in the extra-embryonic ectoderm (Figure 4F, arrowhead) as well as the parietal extra-emrbyonic endoderm (PE) and visceral extraembryonic endoderm (VE, Figure 4F, arrow and open arrowhead, respectively). In contrast, CUL4A levels were ubiquitously low throughout the embryo (Figure $4 \mathrm{G}$ and $4 \mathrm{H})$. At E7.5, CUL4B was mainly detected in the chorionic membrane (Figure 4Ja, arrowhead), which originates partially from the extra-embryonic ectoderm [26]. CUL4B was also detected at high levels in the neuroectoderm (Figure $4 \mathrm{Jb}$, arrow), and at lower levels in other extra-embryonic tissues (Figure 4Jc). Conversely, CUL4A appeared to be present at low levels in all cell types, except for slightly higher expression in the newly formed neural plate (Figure $4 \mathrm{~K}$ and $4 \mathrm{~L}$, arrow). Collectively, CUL4A is expressed at marginal levels post implantation in the extra-embryonic tissues, while CUL4B appears to be the predominant CUL4 form at these embryonic stages. These findings are consistent with the requirement of CUL4B for survival, while CUL4A likely does not accumulate above the threshold level required to sustain CUL4 ubiquitin ligase activity following CUL4B deletion.

Epiblast-specific deletion of floxed Cul $4 b$ prevents embryonic lethality and generates live Cul $4 \mathrm{~b}$ null mice

Given the essential role of extra-embryonic tissues in supporting embryonic development, we hypothesized that the degeneration of extra-embryonic tissues resulting from $C u l 4 b$ deletion may be the cause of death in $C u l 4 b$ null embryos. We employed a conditional knockout sys- 


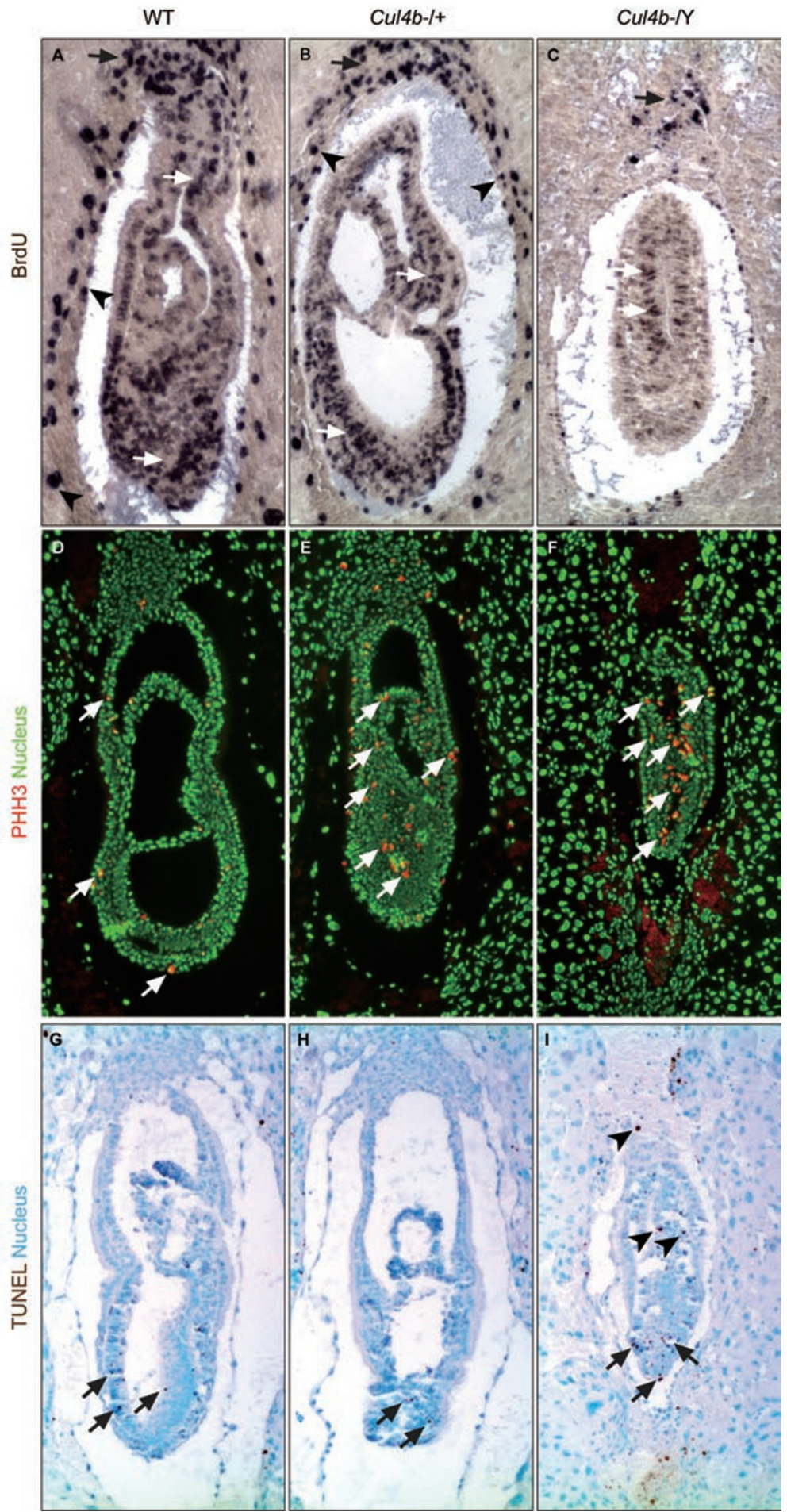

Figure 3 Proliferation and apoptosis status of Cul4b-null embryos. (A-C) BrdU assays revealed many $\mathrm{S}$ phase cells in the ectoplacental cone (black arrows), the parietral extra-embryonic endoderm and underlying endometrium (arrowheads), and in the embryo proper (white arrows) in both wild-type and heterozygous embryos (A, B). Reduction of proliferating cells was detected in those structures in Cul4b-null embryos (C). (D-F) PHH3 IF staining revealed an increase in PHH3-positive cells in the mutant embryos (compare $\mathbf{C}$ to $\mathbf{A}$, arrows). (G-I) TUNEL assays revealed a limited number of apoptotic cells present in either wild-type or heterozygous embryos, primarily in the embryonic ectoderm (G, H, arrows). Dramatic increase in TUNELpositive cells was evident throughout the Cul4b-null embryos (I, arrows). The genotype of individual embryos was determined by immunohistochemical staining with the anti-CUL4B antibody. 


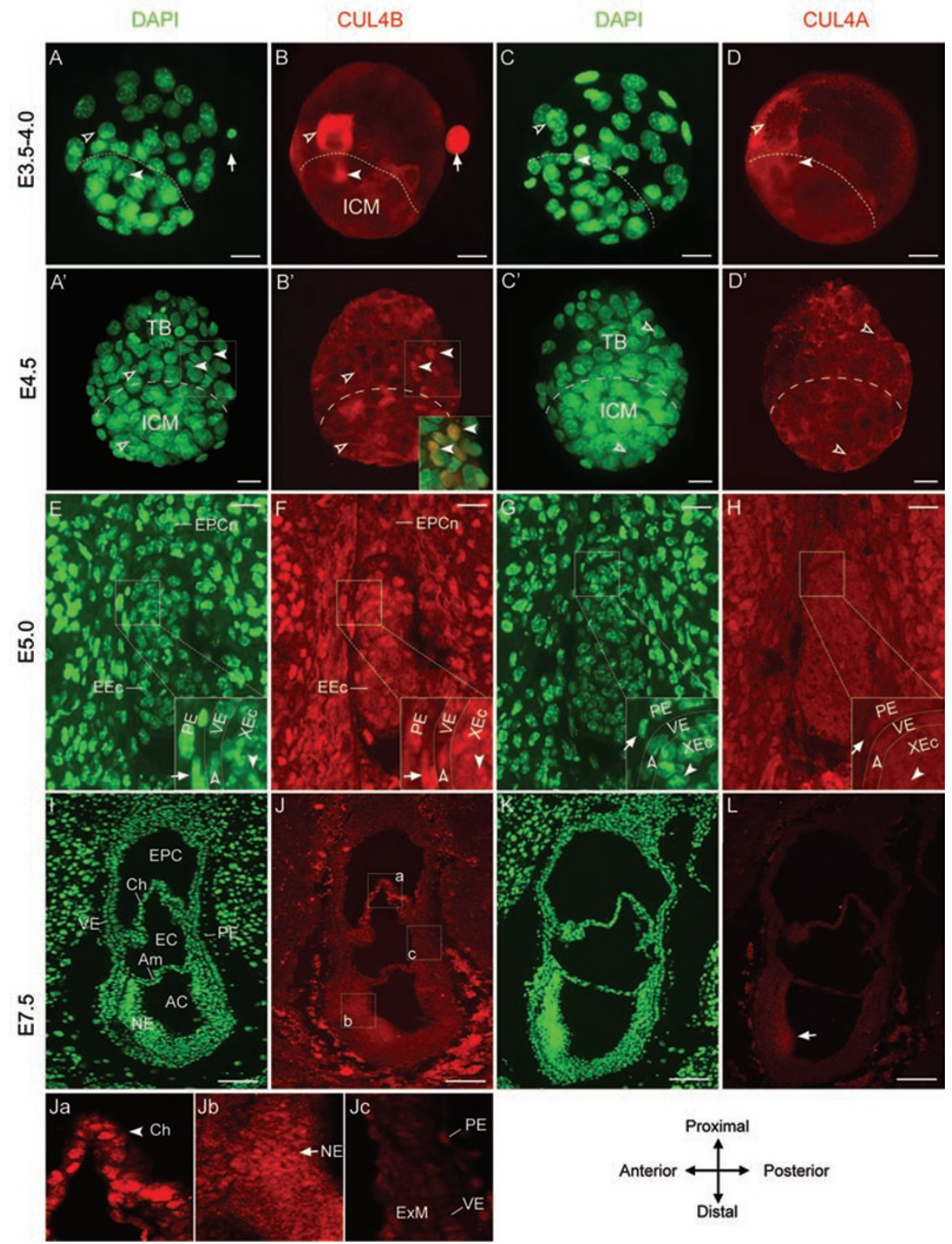

Figure 4 Expression of CUL4A and CUL4B during early embryonic development. (A, B) Immunofluorescent (IF) staining in unhatched (E3.5-4.0) blastocysts revealed expression of CUL4B in the inner cell mass (ICM, arrowheads), the trophoblast (TB, open arrowheads), and the secondary polar body (arrows). (C, D) Low-level expression of CUL4A in the ICM and TB cells. $\left(A^{\prime}-D^{\prime}\right)$ In hatched blastocysts (E4.5), CUL4B was detected in the cytoplasm of most ICM and TB cells (B', open arrowheads), but distinct nuclear staining was evident in some TB cells (B', arrowheads). Inset in $\mathbf{B}^{\prime}$ shows the merged image of the boxed area indicated in $\mathbf{A}^{\prime}$ ' and $\mathbf{B}^{\prime}$ at higher magnification. Arrowheads indicate cells with strong nuclear staining (yellow). At this stage, TB cells are more readily distinguishable from ICM cells as they migrate further away, whereas the latter tend to cluster together. CUL4A, on the other hand, continued to show low cytoplasmic staining in all cells (C', D'). (E, F) At E5.0, CUL4B IF showed prominent expression in the parietal extraembryonic endoderm (PE, arrows), visceral extraembryonic endoderm (VE, open arrowheads) and extra-embryonic ectoderm (XEc, arrowheads). CUL4B is also expressed in the ectoplacental cone (EPCn) and embryonic ectoderm (EEc) at lower levels. (G, H) Weak expression of CUL4A was detected in all tissue layers of E5.0 embryos. (I, J) At E7.5, prominent nuclear staining of CUL4B was evident in the chorionic membrane (Ch, arrowhead in Ja) and neuroectoderm (NE, arrow in Jb). CUL4B was also detected in other tissue layers including PE, VE, and extraembryonic mesoderm (ExM) (Jc). Ja-Jc shows the boxed regions in $\mathbf{J}$ at a higher magnification. EPC, ectoplacental cavity; EC, exocoelomic cavity; AC, amniotic cavity; Am, amniotic membrane. (K, L) Expression of CUL4A was weak throughout the E7.5 embryo with slightly elevated levels in the neuroectoderm (L, arrow). Orientation of E7.5 embryo sections is indicated. Scale bars: $15 \mu \mathrm{m}$ in A-D', $25 \mu \mathrm{m}$ in E-H, $50 \mu \mathrm{m}$ in I, L. 
Table 3 Genotyping analysis of offspring from breedings between Sox2-Cre $e^{+/-}(ð)$ and $C u l 4 b$ mutant ( $(+)$ mice

\begin{tabular}{|c|c|c|c|c|c|}
\hline Breeding & $\mathrm{Cul}^{+b^{+-}}$ & $\mathrm{Cul}_{4 b^{-/ Y}}$ & No. of litters & No. of pups & Average litter size \\
\hline 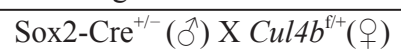 & 4 & 5 & 4 & 31 & 7.8 \\
\hline Sox2-Cre ${ }^{+/-}\left(\bigotimes^{\prime}\right) \times C u l 4 b^{\mathrm{ff}}($ $($ $)$ & 22 & 18 & 10 & 82 & 8.2 \\
\hline
\end{tabular}

A

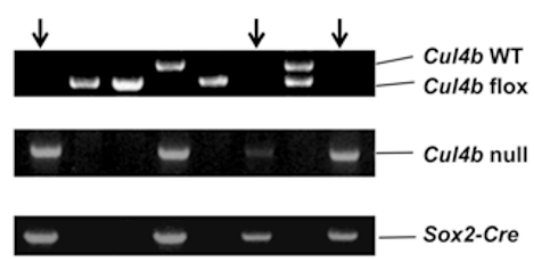

B

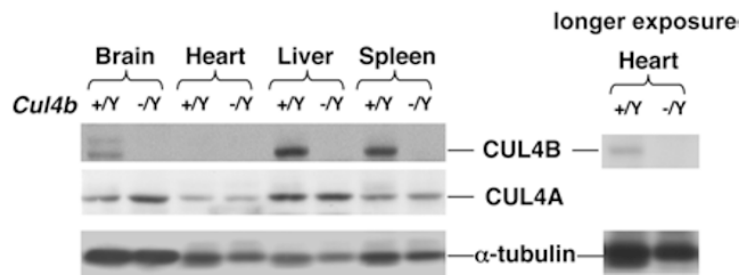

Figure 5 Epiblast-specific deletion of Cul4b gave rise to live Cul4b-null animals. (A) PCR genotyping indicated three $\mathrm{Cul}_{4 b^{f / y}}$ Sox2-Cre animals with Cul4b deletion (marked by arrows). (B) Western blotting to detect Cul $4 b$ deletion in brain, heart, liver, and spleen extracts from $\mathrm{Cul}_{4 b^{-/ \gamma}}$ null mice. Note the comparable expression of Cul4a in wild-type and $\mathrm{Cul} 4 \mathrm{~b}^{-\gamma}$ tissues.

tem in which floxed heterozygous $C u l 4 b^{f /+}$ or homozygous $C u l 4 b^{f f f}$ females were crossed to Sox2-Cre males. Sox2-Cre mice express Cre exclusively in the epiblast [27], and have been utilized previously to circumvent placental defects caused by gene knockout and rescue early embryonic lethality. Indeed, a normal Mendelian ratio of live-born $\mathrm{Cul}_{4} \mathrm{~b}^{-/ \mathrm{Y}}$ null and $\mathrm{Cul} 4 \mathrm{~b}^{+/-}$mice was obtained (Table 3, Figure 5A). These $\mathrm{Cul}_{4} \mathrm{~b}^{-/ \mathrm{Y}}$ mice were healthy and showed no overt developmental abnormalities. Immunoblotting detected no CUL4B protein from several organs of the $C u l 4 b$-null animals, including the brain, heart, liver, and spleen, thus verifying the complete deletion of $C u l 4 b$ in these mice (Figure 5B). CUL4A was detected at comparable or slightly increased levels in the $C u l 4 b$-null organs compared with those in their wildtype littermates. Our results confirm the essential role of Cul4b in the development of extra-embryonic tissues that lack compensatory Cul4a expression (Figure 4). In contrast, $C u l 4 b$ is dispensable for the development of the embryo proper, likely due to the complementing role of
CUL4A that is expressed in the epiblast.

Massive accumulation of the p21 cyclin-dependent kinase inhibitor and cell cycle arrest upon Cul4b inactivation

To gain insight into the molecular mechanisms underlying the developmental defects resulting from $\mathrm{Cul} 4 \mathrm{~b}$ deletion, we examined the protein levels of known CUL4 substrates in an extra-embryonic XEN cell line following $C u l 4 b$ silencing by RNAi. Kunath et al. [28] showed that XEN cells could be reproducibly derived from mouse blastocysts and express lineage markers of the extra-embryonic endoderm. As shown in Figure 6A, p21 protein levels were robustly (16-fold) elevated upon lentiviral shRNA-mediated knockdown of endogenous $C u l 4 b$ in XEN cells. Additional CUL4 substrates were also examined, including SET8 and WDR5, which all remained at similar levels compared to control (scrambled) shRNAexpressing cells. Notably, low levels of CUL4A were detected in Cul4b knockdown XEN cells (Figure 6A), consistent with the IF staining of CUL4A in wild-type extra-embryonic tissues (Figure 4). In agreement with the observed G2 growth arrest of Cul4b $b^{-/ Y}$ embryos (Figure $3 \mathrm{C}$ ), BrdU staining and FACS analysis also indicated a profound $\mathrm{G} 2 / \mathrm{M}$ arrest in Cul4b knockdown XEN cells compared with those infected with scrambled shRNA (Figure 6B, left and middle panels). Cul4b-knockdown XEN cells rapidly ceased to proliferate (Figure 6C). Annexin V staining and FACS analysis also revealed a 5 -fold increase in apoptosis in Cul4b knockdown XEN cells (Supplementary information, Figure S3), recapitulating the increased apoptosis observed in $\mathrm{Cul}_{4} \mathrm{~b}^{-/ Y}$ embryos (Figure 3I). To determine if p21 accumulation contributes to the growth arrest phenotype, we silenced p21 expression by lentiviral shp21 in $C u l 4 b^{k / d}$ XEN cells (Figure 6D). As seen in Figure 6B (right panel), p21 knockdown partially rescued G2 arrest following Cul4b silencing ( $27.1 \%$ vs $48.5 \%$ of G2 population), with resumption of DNA replication ( $43 \%$ vs $19.9 \%$ of S phase population). It is noteworthy that silencing of $\mathrm{p} 21 \mathrm{did}$ not achieve $100 \%$ recovery of G2-arrested $C u l 4 b^{k / d}$ XEN cells as compared to the scrambled shRNA-infected control cells. These results suggest that $\mathrm{p} 21$ is a major but not the sole effector responsible for the proliferation defect in $C u l 4 b^{k / d}$ XEN cells. Consistent with the cell cycle 
A

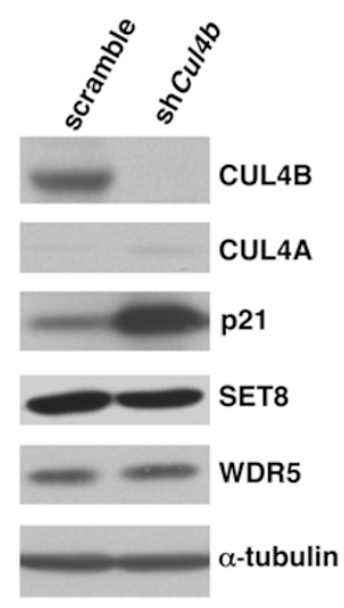

B

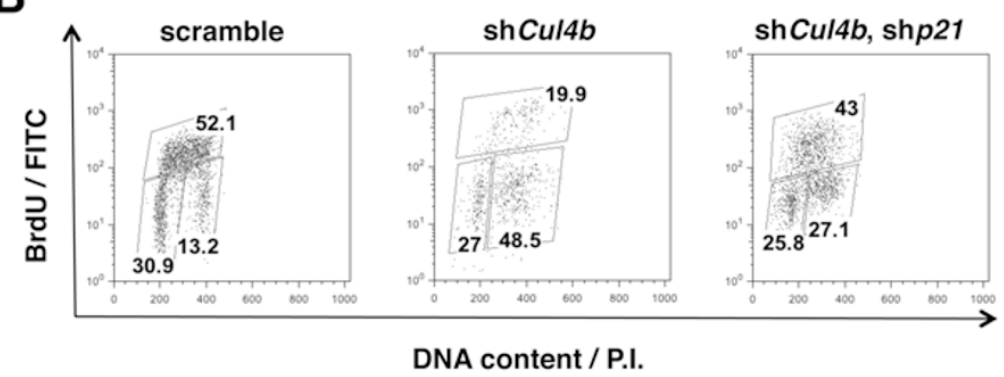

C

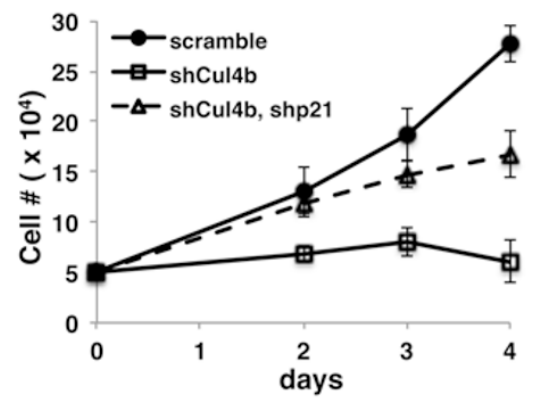

D

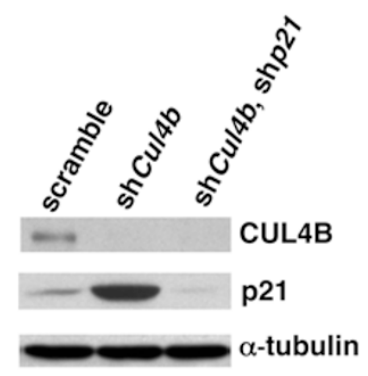

Figure 6 p21 accumulation and G2/M cell cycle arrest upon silencing of Cul4b expression in extra-embryonic XEN cells. (A) XEN cells were infected with lentiviral shCul4b or scrambled shCul4b. CUL4A, CUL4B, and a panel of CUL4 substrates including p21, SET8, and WDR5 were detected by western blotting using the appropriate antibodies. (B-D) G2/M arrest of XEN cells following Cul4b knockdown by lentiviral shRNA and partial rescue by p21 silencing. XEN cells infected with lentiviral scramble shRNA, shCUL4B or both shCUL4B and shp21 were isolated by puromycin selection and FACS. Cells were fixed, stained with anti-BrdU antibody and propidium iodide, and analyzed by flow cytometry (B), counted to determine their growth rates $(\mathbf{C})$, or subjected to western blotting with antibodies against CUL4B, p21, and actin (loading control). The percentages of $\mathrm{G} 1, \mathrm{~S}$, and $\mathrm{G} 2 / \mathrm{M}$ cells in scramble- or shCul4b-expressing XEN cells are indicated.

analysis, silencing of $\mathrm{p} 21$ in $C u l 4 b^{k / d}$ cells overcame the growth arrest and restored cellular proliferation, albeit at a slower rate than in control cells (Figure 6C). We also silenced CUL4A expression by lentiviral shRNA in XEN cells, but observed only a marginal effect on p21 upregulation and no growth inhibition (Supplementary information, Figure S4 and data not shown). Collectively, these results indicate a specific role for CUL4B in the targeted degradation of $\mathrm{p} 21$ in extra-embryonic tissue-derived cells. Further experiments should define the physiological function of CUL4B-mediated p21 destruction in the proliferation and survival of developing extra-embryonic tissues.

\section{Discussion}

The two CUL4 family members have overlapping as well as divergent functions during embryonic development. We previously showed that $\mathrm{Cult}_{4} \mathrm{a}^{-/-}$mice (exons 17-19 deleted) were viable and displayed no developmental abnormalities [12]. Kopanja et al. [13] also showed that another $\mathrm{Cul}_{4} \mathrm{a}^{-/-}$strain (exons 4-8 deleted) was viable and appeared phenotypically normal. Silencing of $\mathrm{Cul} 4 \mathrm{~b}$ expression in primary $\mathrm{Cul} 4 \mathrm{a}^{-/-}$mouse embryonic fibroblasts led to the rapid loss of viability, indicating that CUL4B compensates for the essential functions of CUL4A to support growth and survival [12].

There is currently no evidence that suggests the selective usage of different DCAF substrate receptors by CUL4A or CUL4B, as they both employ the DDB1 adaptor to recruit DCAFs. However, an emerging mechanism that distinguishes the two CUL4 proteins is the nonoverlapping expression pattern of CUL4A and CUL4B in specific tissues and organs during development. For example, $C u l 4 b$ expression is silenced in the pachytenediplotene stages of meiotic prophase, accounting for the reproductive defects and male infertility of the $\mathrm{Cul} 4 \mathrm{a}^{-/}$ mice [14]. Another difference is the predominant cytoplasmic vs nuclear distribution of CUL4A and CUL4B, respectively, suggesting another mechanism of substrate preference between the two CUL4 proteins [29, 30], although a subpopulation of CUL4A also resides in the 
nucleus to target transcription factors and nucleotide excision repair proteins (reviewed in [6-8]). Moreover, in response to environmental toxins, the extreme $\mathrm{N}$ terminus of CUL4B binds to the aryl hydrocarbon receptor (AhR) to form a distinct E3 ligase that catalyzes the ubiquitination and degradation of the estrogen receptor, progesterone receptor, and AhR itself [15].

Here, we showed that the differential expression of CUL4A and CUL4B in extra-embryonic tissues underlies the functional divergence of the two CUL4 proteins. $C u l 4 b^{-/ Y}$ embryos suffer lethality during early postimplantation development due to defects in extra-embryonic tissues, which express low levels of $\mathrm{Cul} 4 \mathrm{a}$ (Figure 4). Deletion of $C u l 4 b$ in the $C u l 4 b^{\mathrm{f} / \mathrm{Y}} C A G$-Cre embryos resulted in overall inactivation of CUL4 ubiquitin ligase activity and induction of growth arrest in extra-embryonic tissues, which were subsequently unable to support the viability of the embryo. We further showed that inactivation of $C u l 4 b$ in an extra-embryonic cell line led to the dramatic accumulation of the p21 cyclin-dependent kinase inhibitor and G2/M arrest (Figure 6). In contrast, the epiblast expresses both $\mathrm{Cul} 4 \mathrm{a}$ and $\mathrm{Cul}$ bb (Figure 4), and, as a result, $\mathrm{Cul} 4 \mathrm{a}$ compensates for the loss of $\mathrm{Cul} 4 \mathrm{~b}$ to maintain viability of the embryo proper. In support of the notion that the differential expression of $\mathrm{Cul} 4 a$ and $C u l 4 b$ in the extra-embryonic tissues is the underlying cause of lethality in $C u l 4 b^{-/ Y}$ embryos, $C u l 4 b^{\mathrm{f} / \mathrm{Y}}$ or $C u l 4 b^{\mathrm{f} /}$ ${ }^{\mathrm{f}}$ mice crossed with Sox2-Cre are viable and develop to adulthood without overt phenotypic deficiencies. However, detailed neurological, behavioral and reproductive analyses have yet to be carried out. Previous studies demonstrated robust ubiquitin-dependent proteolysis in the trophoblast but not the inner cell mass of blastocysts, suggesting a critical role for proteolysis in the extraembryonic tissue development in mice [31]. Our results support this notion by further revealing the essential role of the CUL4B ubiquitin ligase in the extra-embryonic tissues, but not in the epiblast where there is a functional overlap of co-expressed CUL4A and CUL4B.

It is important to note that the $C u l 4 b$ null allele cannot be passed from a heterozygous mother to its male offspring due to paternal X-chromosome inactivation (imprinting) in the extra-embryonic tissues, as evidenced by the absence of $\mathrm{Cul}_{4} b^{+-}$mice from the CAG-Cre (male) x $\mathrm{Cul}_{4} b^{\mathrm{f} / \mathrm{+}}$ (female) mating (Table 1). Consistent with the defects found in the extra-embryonic tissues of the $C u l 4 b^{+/-}$embryos, there was also a lack of vasculature in the mutant yolk sacs compared to the well-organized yolk sac vasculature in wild-type $C u l 4 b^{f /+}$ embryos (Figure 2A). Since murine yolk sac hematopoiesis begins on E7.5 with the migration of hemangioblast precursors from the primitive streak into the mesodermal layer of the visceral yolk sac [32], defects in the mutant yolk sac vasculature are likely secondary, and result from the defective interaction between the mutant visceral endoderm and hemangioblasts. Heterozygous animals were derived by mating male $C u l 4 b^{f / Y}$ with female $C A G$-Cre (Table 2) to ensure that the wild-type $C u l 4 b$ allele is maternally inherited. In contrast, X-inactivation in human extraembryonic placental tissue occurs randomly and the paternal X-chromosome can remain active in a $C U L 4 B$ heterozygous child [33]. Thus, the mutated $C U L 4 B$ allele can be maternally transmitted to the next generation in humans.

To understand the cellular pathways that are deregulated upon $C u l 4 b$ ablation in extra-embryonic tissues, we silenced $C u l 4 b$ expression with lentiviral shRNA in XEN cells and observed rapid growth arrest at G2/M phase. This cell cycle arrest corresponded with a 16-fold increase in p21 protein levels, while levels of several other CUL4 substrates were not affected (Figure 6A). Unfortunately, we were unable to detect p21 specifically by immunohistochemistry in $C u l 4 b^{-/ Y}$ mouse extra-embryonic tissues using commercially available anti-p21 antibodies as cross-reacting signals were observed in the control p $21^{-/-}$specimens (also personal communications with Andrew Koff, Memorial Sloan-Kettering Cancer Center). Previous studies on G2 arrest induced by DNA damage or mitogen starvation revealed that $\mathrm{p} 21$ directly interacts with cyclin A- and cyclin B-associated CDK1 kinase to block G2/M progression [34-37]. Consistent with these studies, accumulation of high levels of $\mathrm{p} 21$ following the depletion of the CUL4B ubiquitin ligase led to growth arrest at G2 phase in the extra-embryonic lineages. ShRNA-mediated silencing of p21 largely rescued the G2 arrest of $C u l 4 b$-knockdown XEN cells and resumed cell proliferation, indicating that $\mathrm{p} 21$ is indeed a major target of CUL4B. However, the inability to achieve full rescue by $\mathrm{p} 21$ silencing suggests that other yet-to-be identified CUL4B substrates likely also contribute to the cell cycle arrest that is triggered by CUL4B inactivation. Spruce et al. [38] recently demonstrated that inhibition of p21 expression by miRNA was required to maintain the trophoblast stem cell compartment. Our findings suggest that p21 accumulation is a significant factor that contributes to the developmental defects of the extra-embryonic tissues and embryonic lethality of $C u l 4 b$-null mice.

Human CUL4B patients survive to adulthood with multiple neuronal and developmental defects. Although $\mathrm{Cul}_{4} b^{-/ \mathrm{Y}}$ mice derived from the Cul4b $b^{\mathrm{f} / \mathrm{Y}} \times$ Sox2-Cre cross can survive with no overt developmental abnormalities, neurological and behavioral analyses have yet to be performed on these mice. We posit that germline Cul4bdeficient mice may model XLMR in patients, although 
the different epigenetic regulations between humans and mice may limit the recapitulation of developmental defects observed in CUL4B patients. Future studies should compare the temporal and spatial expression profiles of human and mouse CUL4A and CUL4B during development and organogenesis, especially in the affected tissues and organs of $C U L 4 B$ XLMR patients. Moreover, the comprehensive proteomic profiling of accumulated substrates following $C u l 4 b$ abrogation could potentially lead to the delineation of cellular pathways that contribute to the clinical symptoms of XLMR with CUL4B mutations, and offer insight into potential therapeutic strategies. Our study elucidates the roles of CUL4B during embryonic development and provides a strategy to generate viable $C u l 4 b^{-/ Y}$ animals, thus setting the stage to probe the physiological and pathological functions of the CUL4B ubiquitin ligase.

\section{Materials and Methods}

\section{Cells, plasmids, and antibodies}

XEN cells were a kind gift from Dr Sundeep Kalantry (University of Michigan, USA). Lentiviruses encoding shCul4b, shCul4a, or scrambled shCul $4 b$ control were generated for infection of XEN cells and were described previously [12]. Antibodies purchased from commercial sources include those against CUL4A (Bethyl Laboratories), CUL4B (Proteintech group), PHH3 (Millipore), p21 (Santa Cruz), WDR5 (R\&D Systems), SET8 (Cell Signaling), and $\alpha$-tubulin (Sigma Aldrich).

\section{Generation of floxed Cul4b mice}

To generate the floxed $C u l 4 b$ allele, a murine $C u l 4 b$ BAC clone was used to generate the final targeting construct. Targeted disruption of the Cul $4 b$ locus was accomplished by placing a LoxP site and an $X b a$ I cutting site 135 bp upstream of exon 4 and another LoxP site and the neomycin resistance cassette $66 \mathrm{bp}$ downstream of exon 5. The $5^{\prime}$ and $3^{\prime}$ homologous arms were $4.3 \mathrm{~kb}$ and $4 \mathrm{~kb}$, respectively. Following electroporation and G418 selection, clones that underwent homologous recombination were identified by Southern blotting after $\mathrm{Xba \textrm {I }}$ digestion using a 5 '-flanking probe. Cul $4 b$-targeted mouse ES cells were injected into blastocysts to generate chimeras, from which germline transmission of the targeted Cul4b allele was accomplished and verified by PCR (Supplementary information, Data S1). The deleted Cul $4 b$ allele was created by germline-induced deletion of exons 4 and 5 by crossing floxed Cul $4 b$ mice with the CAG-Cre or SOX2-Cre transgenic lines.

Histology, immunofluorescence, and immunohistochemistry

Embryos were collected from timed pregnancy at various stages of gestation for further analysis. Fresh deciduas were fixed in $4 \%$ paraformaldehyde overnight at $4{ }^{\circ} \mathrm{C}$, dehydrated and embedded in paraffin. Whole mount IF on blastocysts was performed as previously described [39]. Standard IF was performed on PFA-fixed paraffin sections $(6 \mu \mathrm{m})($ E5.0) or frozen sections (E7.5) following procedures as previously described [40]. Immunohistochemistry was performed on paraffin-embedded embryo sections as previously described in detail [12].

Proliferation and TUNEL assay

7.5 d.p.c. pregnant female mice were pulse-labeled via intraperitoneal injection of $\mathrm{BrdU}$ solution (100 $\mu \mathrm{g}$ BrdU per gram body weight) $1 \mathrm{~h}$ prior to embryo harvest. BrdU assays were performed on PFA-fixed, paraffin-embedded 10-mm sections using BrdU Labeling and Detection Kit (Roche Applied Science, Indianapolis, IN, USA) following the manufacturer's instructions. TUNEL assay were performed on paraffin sections using the TACS ${ }^{\circledR} 2$ TdT-DAB In Situ Apoptosis Detection Kit (Trevigen, Gaithersburg, MD, USA).

\section{Acknowledgments}

We thank Sundeep Kalantry (University of Michigan, USA) for the XEN cells, Wei Gu (Columbia Universtity, USA) for help on generation of the germline-transmitted $C u l 4 b^{f /+}$ mice and Willie Mark (Sloan-Kettering Cancer Center, USA) for the CAG-Cre transgenic mice, and Jennifer Lee and Brent Bany for critical reading of the manuscript. This work is supported by National Institutes of Health Grants CA098210 and CA118085 to PZ, ES016597 to LM, and GM58726 and CA08748 to EL. PZ is supported in part by the Irma T Hirschl Career Scientist Award and the TriInstitutional Stem Cell Initiative. EL is also supported in part by NYSTEM (CO2433).

\section{References}

1 Rejeb I, Ben Jemaa L, Chaabouni H. X linked mental retardation. Tunis Med 2009; 87:311-318.

2 Tarpey PS, Raymond FL, O'Meara S, et al. Mutations in CUL4B, which encodes a ubiquitin E3 ligase subunit, cause an X-linked mental retardation syndrome associated with aggressive outbursts, seizures, relative macrocephaly, central obesity, hypogonadism, pes cavus, and tremor. Am J Hum Genet 2007; 80:345-352.

3 Zou Y, Liu Q, Chen B, et al. Mutation in CUL4B, which encodes a member of cullin-RING ubiquitin ligase complex, causes X-linked mental retardation. A J Hum Genet 2007; 80:561-566.

4 Isidor B, Pichon O, Baron S, David A, Le Caignec C. Deletion of the CUL4B gene in a boy with mental retardation, minor facial anomalies, short stature, hypogonadism, and ataxia. Am J Med Genet A 2010; 152A:175-180.

5 Badura-Stronka M, Jamsheer A, Materna-Kiryluk A, et al. A novel nonsense mutation in CUL4B gene in three brothers with X-linked mental retardation syndrome. Clin Genet 2009; 77:141-144.

6 Lee J, Zhou P. Cullins and Cancer. Genes Cancer 2010; 1:690-699.

7 Sarikas A, Hartmann T, Pan ZQ. The cullin protein family. Genome Biol 2011; 12:220.

8 Jackson S, Xiong Y. CRL4s: the CUL4-RING E3 ubiquitin ligases. Trends Biochem Sci 2009; 34:562-570.

9 Lee J, Zhou P. DCAFs, the missing link of the CUL4-DDB1 ubiquitin ligase. Mol Cell 2007; 26:775-780.

10 Abbas T, Sivaprasad U, Terai K, et al. PCNA-dependent regu- 
lation of p21 ubiquitylation and degradation via the CRL4 ${ }^{\mathrm{Cd} 2}$ ubiquitin ligase complex. Genes Dev 2008; 22:2496-2506.

11 Kim Y, Starostina NG, Kipreos ET. The CRL4 ${ }^{\mathrm{Cd} 2}$ ubiquitin ligase targets the degradation of $\mathrm{p} 21^{\mathrm{Cipl}}$ to control replication licensing. Genes Dev 2008; 22:2507-2519.

12 Liu L, Lee S, Zhang J, et al. CUL4A abrogation augments DNA damage response and protection against skin carcinogenesis. Mol Cell 2009; 34:451-460.

13 Kopanja D, Roy N, Stoyanova T, et al. Cul4A is essential for spermatogenesis and male fertility. Dev Biol 2011; 352:278287.

14 Yin Y, Lin C, Kim ST, et al. The E3 ubiquitin ligase Cullin 4A regulates meiotic progression in mouse spermatogenesis. Dev Biol 2011; 356:51-62.

15 Ohtake F, Baba A, Takada I, et al. Dioxin receptor is a liganddependent E3 ubiquitin ligase. Nature 2007; 446:562-566.

16 Kerzendorfer C, Whibley A, Carpenter G, et al. Mutations in Cullin 4B result in a human syndrome associated with increased camptothecin-induced topoisomerase I-dependent DNA breaks. Hum Mol Genet 2010; 19:1324-1334.

17 Cox BJ, Vollmer M, Tamplin O, et al. Phenotypic annotation of the mouse X chromosome. Genome Res 2010; 20:11541164.

18 Angers S, Li T, Yi X, et al. Molecular architecture and assembly of the DDB1-CUL4A ubiquitin ligase machinery. Nature 2006; 443:590-593.

19 de Boer J, Williams A, Skavdis G, et al. Transgenic mice with hematopoietic and lymphoid specific expression of Cre. Eur J Immunol 2003; 33:314-325.

20 Lakso M, Pichel JG, Gorman JR, et al. Efficient in vivo manipulation of mouse genomic sequences at the zygote stage. Proc Natl Acad Sci USA 1996; 93:5860-5865.

21 Sakai K, Miyazaki J. A transgenic mouse line that retains Cre recombinase activity in mature oocytes irrespective of the cre transgene transmission. Biochem Biophys Res Commun 1997; 237:318-324.

22 Takagi N, Sasaki M. Preferential inactivation of the paternally derived $\mathrm{X}$ chromosome in the extraembryonic membranes of the mouse. Nature 1975; 256:640-642.

23 Lee JT. Disruption of imprinted X inactivation by parent-oforigin effects at Tsix. Cell 2000; 103:17-27.

24 Sado T, Wang Z, Sasaki H, Li E. Regulation of imprinted Xchromosome inactivation in mice by Tsix. Development 2001 ; 128:1275-1286.

25 Payer B, Lee JT, Namekawa SH. X-inactivation and X-reactivation: epigenetic hallmarks of mammalian reproduction and pluripotent stem cells. Hum Genet 2011; 130:265-280.

26 Pereira PN, Dobreva MP, Graham L, et al. Amnion formation in the mouse embryo: the single amniochorionic fold model. BMC Dev Biol 2011; 11:48.
27 Hayashi S, Lewis P, Pevny L, McMahon AP. Efficient gene modulation in mouse epiblast using a Sox2Cre transgenic mouse strain. Mech Dev 2002; 119 (Suppl 1):S97-S101.

28 Kunath T, Arnaud D, Uy GD, et al. Imprinted X-inactivation in extra-embryonic endoderm cell lines from mouse blastocysts. Development 2005; 132:1649-1661.

29 Nakagawa T, Xiong Y. X-Linked mental retardation gene CUL4B targets ubiquitylation of H3K4 methyltransferase component WDR5 and regulates neuronal gene expression. Mol Cell 2011; 43:381-391.

30 Zou Y, Mi J, Cui J, et al. Characterization of nuclear localization signal in the $\mathrm{N}$ terminus of CUL4B and its essential role in cyclin E degradation and cell cycle progression. $J$ Biol Chem 2009; 284:33320-33332.

31 Sutovsky P, Motlik J, Neuber E, et al. Accumulation of the proteolytic marker peptide ubiquitin in the trophoblast of mammalian blastocysts. Cloning Stem Cells 2001; 3:157-161.

32 Chung YS, Zhang WJ, Arentson E, et al. Lineage analysis of the hemangioblast as defined by FLK1 and SCL expression. Development 2002; 129:5511-5520.

33 van den Berg IM, Galjaard RJ, Laven JS, van Doorninck JH. $\mathrm{XCI}$ in preimplantation mouse and human embryos: first there is remodelling. Hum Genet 2011; 130:203-215.

34 Smits VA, Klompmaker R, Vallenius T, et al. p21 inhibits Thr161 phosphorylation of Cdc2 to enforce the G2 DNA damage checkpoint. J Biol Chem 2000; 275:30638-30643.

35 Baus F, Gire V, Fisher D, Piette J, Dulic V. Permanent cell cycle exit in G2 phase after DNA damage in normal human fibroblasts. EMBO J 2003; 22:3992-4002.

36 Medema RH, Klompmaker R, Smits VA, Rijksen G. p21 ${ }^{\text {wafl }}$ can block cells at two points in the cell cycle, but does not interfere with processive DNA-replication or stress-activated kinases. Oncogene 1998; 16:431-441.

37 Niculescu AB, 3rd, Chen X, Smeets M, et al. Effects of p21(Cip1/Waf1) at both the G1/S and the G2/M cell cycle transitions: $\mathrm{pRb}$ is a critical determinant in blocking DNA replication and in preventing endoreduplication. Mol Cell Biol 1998; 18:629-643.

38 Spruce T, Pernaute B, Di-Gregorio A, et al. An early developmental role for miRNAs in the maintenance of extraembryonic stem cells in the mouse embryo. Dev Cell 2010; 19:207219.

39 Riley JK, Carayannopoulos MO, Wyman AH, Chi M, Moley KH. Phosphatidylinositol 3-kinase activity is critical for glucose metabolism and embryo survival in murine blastocysts. $J$ Biol Chem 2006; 281:6010-6019.

40 Yin Y, Lin C, Ma L. MSX2 promotes vaginal epithelial differentiation and wolffian duct regression and dampens the vaginal response to diethylstilbestrol. Mol Endocrinol 2006; 20:1535-1546.

(Supplementary information is linked to the online version of the paper on the Cell Research website.) 